\title{
Hannah Arendt im Spannungsfeld zwischen Säkularisierung und Sakralisierung ${ }^{1}$
}

Die Moderne hat auf der einen Seite einigen Menschen größere Autonomiespielräume gebracht. Auf der anderen Seite hat sie, so wird immer wieder betont, traditionelle und religiöse Gewissheiten zerstört. ${ }^{2}$ Auf den Mangel an Gewissheiten reagiert die politische Theorie, so Markus Holzinger, entweder mit einer "wie auch immer begründeten moralischen Codierung « ${ }^{3}$ oder »streng kontingenzorientiert und dezisionistisch " ${ }^{4}$. Hannah Arendt jedoch ist eine Denkerin, welche diese Dichotomie sprengt, denn sie geht der Frage nach, wie unter Anerkennung der Kontingenz einer Entmoralisierung der Gesellschaft entgegengewirkt werden kann. Um diese Frage zu beantworten, übersetzt sie religiöse Begriffe und Gehalte in ihr politisches Denken und lässt damit neue Handlungsmöglichkeiten aufscheinen. Durch die Aneignung von Begriffen mit religiösen Konnotationen wie Offenbarung, Verzeihen oder den Rückgriff auf Erfahrungen wie die der Selbsttranszendenz gelingt es Arendt, ihr politisches Vokabular mit Bedeutungsgehalten zu füllen, welche aus dem Spiel mit dem religiösen und dem politischen Vokabular entstehen.

Arendt kann in die Reihe jener DenkerInnen eingereiht werden, welche, wie Levinas oder Derrida, laut Jürgen Habermas seit der Spätantike wesentliche religiöse Gehalte in die Sprache der Philosophie übersetzt haben. ${ }^{5}$ Diese DenkerInnen legen es nahe, das Verhältnis von Politik und Religion nicht nur in historischer Sicht daraufhin zu analysieren, wie das Verhältnis von Religion und Politik war und welche Gewaltpotenziale Religion freisetzte, ${ }^{6}$ sondern auch in Hinsicht auf die Frage, welche religiösen Gehalte bei der gewaltlosen Lösung politischer Herausforderungen von Bedeutung sein könnten. Allerdings möchte ich zeigen, dass es sich in

1 Dieser Aufsatz ist aus einem Vortrag im Rhein-Main-Kolloquium »Religion und Politik « entstanden. Ich danke seinen Mitgliedern für ihre hilfreichen Anmerkungen und die Möglichkeit, dort vorzutragen. Weiterhin gilt mein Dank Michael Haus und Gerhard Göhler, welche mich zuverlässig mit konstruktiver Kritik, hilfreichem Rat und Denkanstößen auch beim Entstehen dieses Aufsatzes begleitet haben, sowie den GutachterInnen des Leviathan, deren Bitte um werksgeschichtliche Einbettung sowohl der agonalen Lesart als auch des Aneignungsbegriffs ich hier nicht nachkommen konnte. Aber ich verspreche, es an einem anderen Ort zu tun.

2 Vgl. Greven 1999.

3 Holzinger 2007, S. 83.

4 Ebd.

5 Vgl. Habermas 2012, S. 102.

6 Siehe hierzu Gotthard 2002; Schilling 2007; Burkhardt 2009. 
Arendts Schriften um einen kreativen Aneignungsprozess handelt, welcher die religiösen Bedeutungsgehalte (auf eine für Traditionalisten sicherlich unzumutbar provokative Weise) dem Primat des Politischen unterordnet. Sie zeigt uns, wie wir religiöse Erfahrungen und Praktiken umdeuten und so politisch fruchtbar machen können, um der für unsere Zeit so typischen zynischen Entmoralisierung zu entgehen.

Grundlage für Arendts Antwort auf das Problem des Mangels an Gewissheiten ist ihr Menschenbild, das den Menschen als ein Wesen beschreibt, das sich von einer Gottesvorstellung dadurch unabhängig macht, indem es sich zunehmend göttliche Fähigkeiten wie das Erschaffen von etwas Neuem oder die Fähigkeit zum Verzeihen aneignet. Obwohl sie dieses Verständnis vom Menschen in die Nähe von Nietzsches nihilistischem Denken rückt, verfällt Arendt jedoch nicht in Nietzsches Absage an Moral und Ethik, sondern verweist mit ihrer Handlungstheorie auf das intersubjektive Element und den Möglichkeitshorizont, welcher das gemeinsame Handeln eröffnet. Flankiert wird Arendts Menschenbild von einer Säkularisierung der Erfahrung von Selbsttranszendenz ${ }^{7}$ und einer Sakralisierung ${ }^{8}$ des menschlichen Lebens. Arendt, so kann das Argument zugespitzt werden, arbeitet an der Entstehung von Werten, indem sie Praktiken wie das Erschaffen, Verzeihen, Sakralisieren aus dem religiösen Zusammenhang löst und radikal politisch deutet. Anders als Hans Joas, der mit der Sakralisierung der Person nach einer Möglichkeit sucht, den Schutz der Person dem Politischen zu entziehen und ihr via Heiligkeit einen unverfügbaren Charakter zu geben, bleibt die Heiligkeit der Person bei Arendt damit immer dem Spiel der Kontingenz ausgesetzt und von Aushandlungen abhängig. ${ }^{9}$

Es geht mir darum, zu zeigen, dass Arbeiten, Herstellen und Handeln als Entwicklungsschritte im Emanzipationsprozess des Menschen gedeutet werden können. Und dass die in sämtlichen von Arendt beschriebenen Aneignungsprozessen sichtbar werdende kreative Kraft des Menschen schließlich von Arendt durch zwei theoretische Weichenstellungen gezügelt wird - die Säkularisierung der Erfahrung der Selbsttranszendenz und die Sakralisierung der Person.

\section{Das Erreichen der Unabhängigkeit}

In ihrer Doktorarbeit »Der Liebesbegriff bei Augustin «10 schreibt Arendt 1929:

7 Hier beziehe ich mich auf Joas' Verständnis des Begriffs, welcher Selbsttranszendenz unter anderem als das Gefühl des Ergriffenseins und die Erfahrung des Einsatzes für andere Menschen oder Werte versteht. Vgl. Joas 1999, S. 285.

8 Zur neu aufflammenden Diskussion über das Heilige in der Moderne vgl. auch Canal et al. 2013, S. 7.

9 Hans-Martin Schönherr-Mann macht aber in seiner Einleitung zu Arendt zu Recht darauf aufmerksam, dass nicht alle wesentlichen politischen Begriffe Arendts aus der religiösen Tradition stammen. Vgl. Schönherr-Mann 2006.

$10 \mathrm{Zu}$ den Begriffen der Weltentfremdung und Weltlosigkeit in Arendts Dissertation vgl. Frank 2001. 
»In der auf Adam gegründeten societas hat der Mensch sich unabhängig gemacht von seinem Creator. Er ist auf andere Menschen angewiesen, nicht auf Gott. Das genus humanum hat seinen Ursprung in Adam und nicht im Creator. Es ist entstanden generatione und hat den Bezug zu seinem Ursprung nur durch alle Generationen hindurch. Die Gemeinschaft der Menschen, gegründet auf ihre Verwandtschaft, ist also eine societas von den Toten her und mit den Toten. Das aber heißt, sie ist geschichtlich. Die Unabhängigkeit dieser Welt von Gott gründet gerade in dieser Geschichtlichkeit. Ihre Sündigkeit ist gerade der eigene, von Gott unabhängige Ursprung. «11

Der Mensch ist durch den Sündenfall nun nicht mehr auf Gott, sondern auf andere Menschen angewiesen. Er hat jetzt die Verantwortung für seine Freiheit und damit die Aufgabe, mit der menschlichen Pluralität ohne den Rückbezug auf einen allmächtigen Gott ${ }^{12}$ umzugehen. Die Emanzipation von Gott ist mit dem Sündenfall aber noch nicht beendet, sondern durchläuft in den Arbeiten Arendts drei weitere Stadien, welche jedoch nicht als abgeschlossene Stadien zu verstehen sind, sondern eher als gleichzeitig nebeneinander bestehende und sich überlappende Stadien, die sich aber gleichwohl in einer historischen Reihenfolge herausgebildet haben.

Im Arbeitsprozess ist der Mensch mittels der körperlichen Notwendigkeiten noch in den Kreislauf der von Gott geschaffenen Natur eingebunden, im Prozess des Herstellens erschafft der Mensch neue Dinge, aber er muss dabei auf die Natur zurückgreifen, um das Material für seine Kreationen zu gewinnen. Erst im MiteinanderSprechen und -Handeln kann sich der Mensch von Gott völlig emanzipieren, weil es ihm hier möglich ist, allein im intersubjektiven Zwischenbereich seine Wirklichkeit zu erschaffen, ohne Rückgriff auf Hilfsmittel aus der Natur. ${ }^{13}$

Zum Arbeitsprozess schreibt Arendt:

»Was der biologische Körperprozess und der weltliche Prozess von Wachstum und Verfall miteinander gemein haben, ist, dass beide Teile des Kreislaufs der Natur sind und infolgedessen in unendlicher Wiederholung auftreten. Menschliche Tätigkeiten, die der Notwendigkeit entspringen [...] sind daher selbst in den Kreislauf der Natur gebunden ", dessen »Mühe und Plage « erst mit dem Tod des jeweiligen Organismus ein Ende findet. ${ }^{14}$

Das Arbeiten entspringt dem Gebundensein des menschlichen Körpers an die organischen Notwendigkeiten. Es wird durch die Notwendigkeit, dem Körper Nahrung zuzuführen, erzwungen. Der Mensch kann sich von den organischen Notwendigkeiten nur durch den Tod befreien und erfährt dadurch sein Gebundensein an die Natur und den Schöpfer derselben selbst noch nach dem Sündenfall.

Mit dem Herstellen jedoch gelingt dem Menschen ein großer emanzipatorischer Schritt, denn er beginnt selbst seine Welt zu erschaffen. Zwar ist er im Herstellungsprozess der Dingwelt noch auf das von Gott geschaffene Material angewiesen

11 Arendt 2003 [1929], S. 115.

12 Allmächtigkeit, so Arendt, zielt immer auf die Vernichtung von Pluralität. Vgl. Arendt 2007 [1960].

13 Abgesehen von der Naturhaftigkeit des menschlichen Körpers selbst.

14 Arendt 2007 [1960], S. 117. 
und muss darauf zurückgreifen, aber er wird zum Schöpfer einer ganz eigenen Dingwelt.

»Das Werk unserer Hände, und nicht die Arbeit unseres Körpers, Homo faber, der vorgegebenes Material bearbeitet zum Zwecke der Herstellung, und nicht das Animal laborans, das sich körperlich mit dem Material seiner Arbeit >vermischt` und ihr Resultat sich einverleibt, verfertigt die schier endlose Vielfalt von Dingen, deren Gesamtsumme sich zu der von Menschen erbauten Welt zusammenfügt. « ${ }^{15}$

Im Sprechen und Handeln schließlich erreicht der Mensch die größte Unabhängigkeit von Gott, indem er seine eigene Realität erschafft. Der Mensch wird selbst zum Schöpfer seiner Realität, indem er sich seiner Freiheit bewusst wird und diese verantwortungsvoll und unter Berücksichtigung der Unvorhersehbarkeit der Folgen des Handelns nutzt. Das Wunder der Freiheit liegt für Arendt im kreativen Neubeginn, im Anfangen-Können. ${ }^{16}$

"Sprechend und handelnd schalten wir uns in die Welt der Menschen ein, die existiert, bevor wir in sie geboren wurden, und diese Einschaltung ist wie eine zweite Geburt, in der wir die nackte Tatsache des Geborenseins bestätigen, gleichsam die Verantwortung dafür auf uns nehmen. Aber wiewohl niemand sich diesem Minimum an Initiative ganz und gar entziehen kann, so wird sie doch nicht von irgendeiner Notwendigkeit erzwungen wie das Arbeiten, und sie wird auch nicht aus uns gleichsam hervorgelockt durch den Antrieb der Leistung und die Aussicht auf Nutzen " ${ }^{17}$ wie beim Herstellen.

Die Wirklichkeit entsteht nach Arendt im öffentlichen Miteinander-Sprechen und -Handeln, unabhängig von Gott. Beeinflusst werden kann sie nur durch die menschliche Art der Interaktion. Das heißt, sie kann wie eine Dingwelt mit Blick auf den Nutzen hergestellt werden, oder aber sie kann als Möglichkeitsraum für die Entstehung einer sozialen Welt genutzt werden.

Arendts Postulat der Unabhängigkeitserklärung des Menschen gegenüber Gott unterscheidet sich von Nietzsches Postulat vom Tode Gottes insofern, als dass Gottes Existenz nicht negiert wird, sondern sich der Mensch von Gott geistig emanzipiert, zugleich aber mittels der Notwendigkeiten des Körpers an die Natur gebunden bleibt. Der Mensch ist zwar noch durch natürliche und damit von Gott geschaffene Notwendigkeiten bestimmt, er ist aber in seiner weiteren Entwicklung darüber hinaus nicht mehr auf Gott angewiesen. Er wird selbst zum Schöpfer der Dingwelt und, noch viel wichtiger, zum Schöpfer geteilter Sinngehalte und damit zum Schöpfer der sozialen Wirklichkeit, in der er lebt. Ob Gott in dieser Welt noch eine Rolle spielt und welche, darüber entscheidet allein der Mensch.

Arendt unterscheidet sich von Nietzsche aber auch darin, dass sie danach nicht zu einer Feier des Willens zur Macht, ${ }^{18}$ verstanden als Herrschaft über die Welt und andere, übergeht, sondern dass sie die Unabhängigkeitserklärung gegenüber Gott einerseits mit einem normativ aufgeladenen Begriff des Handelns verknüpft und andererseits mit einer Sakralisierung des menschlichen Lebens sowie einer Säkularisierung der Erfahrung von Selbsttranszendenz.

\author{
15 Ebd., S. 161. \\ 16 Vgl. Arendt 2007 [1993]. \\ 17 Arendt 2007 [1960], S. 215. \\ 18 Vgl. De La Rosa 2012.
}

Leviathan, 42. Jg., 2/2014 


\section{Die kreative Kraft des Menschen}

Indem sich der Mensch von Gott emanzipiert, geht auch die Fähigkeit der Neuschöpfung auf ihn über. Der Mensch erschafft sich seine Welt. Er kann dies als Homo faber tun, die Politik der Notwendigkeit ${ }^{19}$ betreiben und das Politische auf Herrschaftsaspekte beschränken, oder - und das ist die Alternative zu Nietzsche, die Arendt uns aufzeigt - er kann die Politik der Freiheit ${ }^{20}$ verfolgen und das Politische als den Ort sehen, wo die Menschen in Freiheit und wechselseitiger Anerkennung ihrer Einzigartigkeit die Welt gestalten. Dazu ist es aber notwendig, dass die Menschen der im Miteinander-Sprechen und -Handeln angelegten »Rationalität « folgen, welche Arendt mit ihren Konzepten des Handelns, der kommunikativen Macht und des Urteilens zu artikulieren suchte. Habermas hat versucht, diese Rationalität als die Rationalität der guten Gründe zu deuten; ${ }^{21}$ ein anderer Strang der Arendt-Rezeption, vertreten etwa durch Dana Villa ${ }^{22}$ und Amy Allen ${ }^{23}$, versucht dagegen die agonalen und performativen Elemente in Arendts Schriften herauszustellen und deren Rationalität stark zu machen. Die agonale Lesart von Dana Villa arbeitet zwar wichtige Elemente der agonalen Rationalität heraus, sie ist aber zugleich nicht in der Lage, einen normativen Maßstab für die Einhegung des Agons $^{24}$ zu liefern, weil sie diesen im Konzept des Urteilens sucht.

Die performativen ${ }^{25}$ und agonalen Elemente sind vor allem in der Vita activa unübersehbar. Der öffentliche Bereich wird zum "Erscheinungsraum ", eine Art Bühne, auf welcher politische Akteure in Erscheinung treten können und in einem Wettkampf zeigen können, wer sie sind. Dazu benötigen sie aber auch ein entsprechendes Publikum, welches über das Gesehene und Gehörte urteilt. Erst im Zusammenspiel der politisch Handelnden miteinander und mit dem Publikum entsteht kommunikative Macht, entsteht die menschliche Wirklichkeit, entfaltet sich die agonale Rationalität. ${ }^{26}$ Im Herzen dieses Modells findet sich jedoch kein normativer Maßstab, welcher zu unterscheiden hilft, welche Art der Performanz im Wettkampf auf der politischen Bühne die wertvollere oder bessere wäre, sondern lediglich ein Ethos, welches die Akteure dazu anhält, sich mehr auf das Spiel selbst als aufs Gewinnen zu konzentrieren. ${ }^{27}$ Villa weist daher zu Recht darauf hin, dass Arendt

19 Vgl. Arendt 2007 [1993].

20 Vgl. ebd.

21 Vgl. Habermas 1995; Habermas 1991.

22 Villa 1992.

23 Allen 1999.

24 Agon steht in der griechischen Klassik für einen friedlichen Wettkampf.

25 Der Begriff der Performanz steht in der Performanzforschung für den Prozess der Übermittlung und Generierung von Bedeutungsgehalten durch das Handeln. Vgl. Alexander 2011, S. 82.

26 Vgl. Arendt 2007 [1960].

27 Vgl. Villa 1992. 
an diesem Punkt sehr nahe an Nietzsche heranrückt, wenn sie vor allem das Erscheinen von Bedeutung im performativen Akt, also im Wettkampf feiert.

Normativ eingehegt wird diese Feier des Erscheinens, so Villa, jedoch durch das Konzept des Urteilens, in welchem Arendt im Rückgriff auf Kant ein deliberatives Element einführt, denn es ist das Publikum, welches aus einer gewissen Distanz heraus über den Wettkampf urteilt.

»Das politische Urteil, betrachtet als eine Art Geschmacksurteil, hilft jedenfalls die Kämpfenden zu bändigen, indem es die Verbindung von Pluralität und Überlegung wieder einführt, und zeigt, wie die Urteilsaktivität möglicherweise im Prozess der Artikulation ihrer Differenzen einem Publikum eröffnet, was es miteinander gemein hat. « ${ }^{28}$

Allein durch die Artikulation von Differenz werden jedoch noch keine Gemeinsamkeiten sichtbar, und auch die Aktivität des Urteilens an sich birgt noch keinen normativen Maßstab. Villa kann daher nicht überzeugend zeigen, wie das Konzept des Urteilens von Arendt einen normativen Maßstab zur Verfügung stellen kann, welcher die Spielregeln für den agonalen Wettkampf stellt. Das wird deutlich, wenn man Arendts Konzept des Umgangs mit der Vergangenheit und des Urteilens auf den Begriff der Aneignung hin befragt. Dann wird sichtbar, dass sowohl Arendts Konzept der Geschichtsschreibung als auch ihr Konzept des Urteilens auf der Vorstellung von einem kreativen Aneignungsprozess beruht, welcher an sich über keinen normativen Maßstab verfügt, das heißt der normative Maßstab muss durch ein anderes Konzept gewonnen werden.

\section{Der aneignende Charakter der Geschichtsschreibung}

Arendt ist wie Nietzsche einer hermeneutischen Tradition zuzurechnen, welche das menschliche Sprechen und Handeln als einen Akt der Aneignung begreift. In »Vom Leben des Geistes " geht Arendt daher der Frage nach, wie der Beobachter der Vergangenheit Bedeutung abgewinnen kann. Es geht ihr also wohlgemerkt nicht darum, der Vergangenheit eine richtige Bedeutung abzugewinnen. Sie stützt sich dabei auf Hegels Konzept der Zeit. Dieser konzipierte eine Philosophie der Geschichte, also der Vergangenheit, welche den Vorgang beschreibt, wie sich das denkende Ich die Vergangenheit aneignet und so Geist und Welt versöhnt:

»Nach Hegel kann der Geist durch die bloße Macht der Reflexion zwar natürlich nicht alle Erscheinungen sich aneignen - gewissermaßen in sich hineinsaugen -, wohl aber alles, was an ihnen sinnvoll ist; und was er sich nicht aneignen kann, lässt er als bedeutungslosen Zufall beiseite, als ohne Konsequenzen für den Geschichtsverlauf oder für das diskursive Denken. «29

Der Vorrang der Vergangenheit verschwindet aber, so Arendt, wenn der Geist beginnt, sich mit der Zukunft zu beschäftigen, denn dann, fährt sie fort, ist es ent-

28 » Yet political judgment seen as a kind of taste judgment nevertheless helps to tame the agon by reintroducing the connection between plurality and deliberation, by showing how the activity of judgment can, potentially, reveal to an audience what they have in common in the process of articulating their differences. (Ebd., S. 298, Übersetzung S.D.L.R.)

29 Arendt 2006 [1979], S. 279.

Leviathan, 42. Jg., 2/2014 
gegen Hegels Annahmen nicht das denkende, sondern das wollende Ich, welches das Vermögen hat, die Gegenwart zu negieren, sie also zu verändern. Der Ablauf der Dinge ist kein vorherbestimmter, sondern kann durch das Vermögen des wollenden Ichs verändert werden. Der Mensch erschafft sich, so Arendt, mittels des Willens, »seines Organs für die Zukunft «, selbst. Der Wille ist zugleich das Organ für die Quelle der Zukunft wie der Vergangenheit, denn die Vergangenheit wird von der Zukunft geschaffen. ${ }^{30}$

Ich deute Arendts Überlegungen zur Geschichtsschreibung so, dass Tradition für Arendt durchaus eine Quelle der Wahrheit ist, jedoch nicht, weil es ein objektives hermeneutisches Verfahren gäbe, welches der Vergangenheit die richtige Bedeutung abgewinnen könnte, sondern weil es gelingt, Neues in die Welt zu holen, indem der Mensch eine Vorstellung von einer möglichen Zukunft entwickelt und eine Geschichte erzählt, welche das Neue in der Vergangenheit verortet, das heißt eine Geschichte erzählt, welche die Fragmente der Vergangenheit zu einem sinnvollen Ganzen zusammenfügt. Das ausführlichste Beispiel hierfür findet sich in Arendts Beschäftigung mit der amerikanischen Revolution. Die narrative Struktur des Handelns und der menschlichen Identität hat für Arendt zur Folge, dass die Vergangenheit immer wieder in die Geschichten der Gegenwart eingeschrieben werden muss. Diese Wiederaneignung der Vergangenheit bedeutet ständige Neueinschätzungen und Neubewertungen der Vergangenheit, welche in manchen Fällen, wie jenem der amerikanischen Revolution, nur außerhalb der Tradition erfolgen kann. Diese Momente des Neubeginns sind jedoch durch eine absolute Beliebigkeit gekennzeichnet: »Jeder Anfang birgt in sich ein Element völliger Willkür «. ${ }^{31}$ Um diese Beliebigkeit zu überwinden, wandten sich die Gründerväter der amerikanischen Verfassung den römischen Institutionen zu, nicht, so Arendt, aus einer romantischen Geste heraus, sondern weil sie ein Modell benötigten, um die politische Ordnung zu stabilisieren. ${ }^{32}$ Deshalb eigneten sie sich das römische Modell an und veränderten es, indem sie die Autorität des Obersten Gerichtshofs aus der geschriebenen Verfassung ableiteten und nicht, wie im römischen Senat, von der Autorität der Gründerväter.

\section{Der aneignende Charakter des Urteilens}

Auch das Konzept der erweiterten Denkungsart basiert auf der Idee der Aneignung. Das Vermögen, das eine Aneignung möglich macht, heißt »Einbildungskraft «.33 Diese Einbildungskraft besteht für Arendt aber nicht in der passiven Übernahme der Vorurteile und Standpunkte anderer, sondern in deren Beurteilung aus der Distanz,

30 Vgl. Arendt 2006 [1979].

31 Vgl. Arendt 2006 [1965], S. 265.

32 Vgl. Arendt 2006 [1965].

33 Arendt 2006 [1979], S. 446. 
welche die innere Repräsentation schafft. Die zeitliche und örtliche Distanz wird somit zu einem notwendigen Element eines guten Urteils.

»Nur was einen in der Vorstellung anrührt, wenn es nicht mehr durch seine unmittelbare Gegenwart anrühren kann [...] nur das lässt sich als recht oder unrecht, wichtig oder unwichtig, schön oder hässlich oder ein Mittelding davon beurteilen. « ${ }^{34}$

Zu dieser Einbildungskraft muss nach Arendt jedoch ein sensus communis, das heißt der gesunde Menschenverstand hinzutreten. Darunter versteht Arendt mit Kant ein Beurteilungsvermögen, welches die Urteile anderer antizipiert:

»Dies geschieht nun dadurch, dass man sein Urteil an anderer nicht sowohl wirkliche, sondern vielmehr bloß mögliche Urteile hält und sich in die Stelle jedes anderen versetzt, indem man bloß von den Beschränkungen, die unserer eigenen Beurteilung zufälligerweise anhängen, abstrahiert. « 35

Die Geltung der so gewonnenen Urteile gleicht jedoch nicht jener kognitiver oder wissenschaftlicher Aussagen, und sie sagt noch nichts darüber aus, wie man handeln soll. ${ }^{36}$ Dieses Urteil des denkenden Ichs ist nicht zwingend, sondern kann nur selbst wiederum um Zustimmung werben. Daher denkt Arendt auch das Publikum als debattierende Instanz, deren Produkt ein immer prekärer, weil durch Diskussionen veränderbarer Common sense darstellt, in welchem sich das Publikum die Welt über gemeinsame Narrationen aneignet.

Als normative Einhegung des agonalen Wettkampfs ist daher der Rückgriff auf das Konzept des Urteilens, wie Villa ihn wählt, nicht ausreichend. Zusammenfassend lässt sich sagen, dass dies daran liegt, dass Arendt sowohl in ihren Überlegungen zur Geschichtsschreibung als auch in ihren Überlegungen zum Urteilen auf den aneignenden Charakter von Geschichtsschreibung und auf den rhetorischen Charakter des Urteilens verweist. Sie sind also beide Gegenstand des rhetorischen Wettkampfs und nicht einhegendes Moment desselben.

Der normative Maßstab findet sich vielmehr in der Trennung von Herstellen und Handeln. Villa übersieht, dass Arendt eine Einschränkung vorgenommen hat, indem sie Herstellen und Handeln trennt und das Handeln als das für den politischen Bereich konstitutive Element setzt. Für Arendt ist das Verhältnis des Menschen zur Vergangenheit und zu anderen Menschen wie für Nietzsche ein aktives und produktives. Anders als Nietzsche bestimmt sie den politischen Wettkampf aber von Anfang an als fairen Wettkampf ${ }^{37}$ zwischen Handelnden, also sich wechselseitig als einzigartig anerkennenden Menschen, und das Urteilen mit seinen beiden Elementen der Einbildungskraft und des sensus communis als dialogischen Prozess. Das Verstehen der Vergangenheit wird so zu einem kreativen und zugleich dialogischumbildenden Akt der Aneignung von Geschichte, nicht wie bei Nietzsche zu einem Akt der Beherrschung. Um das Gesagte auf den Punkt zu bringen: Während Nietzsche nur eine Form der Aneignung von Welt kennt, welche darauf abzielt, den anderen oder das andere zu beherrschen, unterscheidet Arendt zwischen einer Form

34 Ebd., S. 454.

35 Ebd., S. 458.

36 Vgl. ebd.

37 Indem sie das Politische über das Miteinander-Sprechen und -Handeln definiert.

Leviathan, 42. Jg., 2/2014 
der Aneignung, welche darauf abzielt, zu beherrschen, und nennt sie das Herstellen und eine Form der Aneignung von Welt, welche im dialogischen Wettkampf mit den anderen Sprechern unter Anerkennung der Einzigartigkeit jedes Sprechers neue Bedeutungsgehalte entstehen lässt. Das heißt, der politische Wettkampf, den Arendt vor Augen hat, findet nur zwischen Handelnden statt. Es ist also ein Wettkampf, der zwischen Menschen ausgetragen wird, welche sich in ihrer Einzigartigkeit anerkennen und welche auf die Instrumentalisierung des Kontrahenten und seiner Argumente in der Debatte verzichten. Der politische Wettkampf ist so in das normativ gehaltvolle Konzept des Handelns eingebettet. Es ist weniger das Urteilen, welches die Rationalität der Interaktionen sichert, als vielmehr die Haltung gegenüber den am Wettkampf Beteiligten und die Haltung gegenüber denen, die beurteilt werden. Das Politische kann nur dann zu einem Ort werden, wo Menschen in Freiheit ihre Welt gestalten, wenn sie sich wechselseitig das Recht zugestehen, ihre Perspektiven, Erfahrungen und Lösungsansätze vor Publikum zu artikulieren. Die agonale Rationalität liegt darin begründet, dass Konflikte ausgesprochen und ausgetragen werden. Eingehegt werden sie durch die Anerkennung der Legitimität der Forderung, vor Publikum gehört zu werden, und die anerkennende Haltung gegenüber allen am öffentlichen Diskurs Partizipierenden.

Arendt lässt es dabei jedoch noch nicht auf sich bewenden. Sie arbeitet zwei weitere Elemente ein, welche der Einhegung des Agons dienen - sie säkularisiert die Erfahrung der Selbsttranszendenz, und sie sakralisiert das menschliche Leben.

\section{Die Säkularisierung der Erfahrung von Selbsttranszendenz}

Hans Joas unterscheidet in seinen Arbeiten neun verschiedene Formen der Säkularisierung: zwei Formen der Säkularisierung rechtlicher Art, zwei genealogischer Art, drei sozialwissenschaftlich übliche (»Abnahme von Religion; Rückzug der Religion ins Private; Freigabe gesellschaftlicher Bereiche von religiöser Kontrolle « $)^{38}$ und mit Taylor die sogenannte »Säkulare Option « ${ }^{39}$. Die neunte Form ist schließlich die affirmative Genealogie ${ }^{40}$, welche ich als die Aneignung religiöser Elemente (Praktiken und Ideen) mittels Uminterpretation und Eingliederung in den politischen Bereich durch die (politische, soziologische) Theorie bezeichne. Diese Eingliederung nimmt bei Arendt und Joas zwei unterschiedliche Formen an: einmal die Form der Sakralisierung der Person und einmal die Säkularisierung der Gottesbeziehung. Während Joas die Genese einer sozialen Vorstellung von Heiligkeit beschreibt, um diese zugleich zu affirmieren und um die Person in den Status des Heiligen zu erheben und damit zu etwas Außerpolitischem, etwas Unverfügbarem werden zu lassen, verbleibt Arendts Schutz der Person im Bereich des Politischen und damit des Umstrittenen. Als Aneignung religiöser Elemente durch Arendt kann die Uminter-

38 Joas 2009, S. 293.

39 Ebd.

40 Vgl. Joas 2011. 
pretation der Erfahrung von Selbsttranszendenz und die Sakralisierung des Menschen als Praxis der (Selbst-)Heiligung angeführt werden.

\section{Die Uminterpretation der Erfahrung von Selbsttranszendenz}

Während im religiösen Bereich die Erfahrung von Selbsttranszendenz auf eine Erfahrung mit einer göttlichen Instanz gerichtet ist, sich also zwischen dem Göttlichen und dem Gläubigen ereignet, übersetzt Arendt diese Erfahrung in den politischen Bereich, indem sie die Möglichkeit der Erfahrung der Selbsttranszendenz in intersubjektiven Interaktionen aufzeigt und die Rahmenbedingungen beschreibt, welche eine solche Erfahrung ermöglichen. Arendts Konzepte der kommunikativen Macht und des öffentlichen Raums können vor diesem Hintergrund als Beschreibung der Ermöglichungsbedingungen für die Entstehung von Werten, wie Joas sie in Die Sakralisierung der Person umschrieben hat, verstanden werden. Werte entstehen, so Joas, nicht allein dadurch, dass jemand diese als Werte setzt, sondern sie sind von einem »Gefühl der subjektiven Evidenz bei affektiver Intensität « abhängig, das heißt sie müssen erfahren werden. ${ }^{41}$ Die Erfahrbarkeit von Werthaftigkeit und damit auch die Entstehung einer neuen Wirklichkeit ist, wie ich mit Arendt zu zeigen versucht habe, aber von bestimmten Voraussetzungen abhängig, welche ich mit dem Begriff der agonalen Rationalität bezeichnet habe: Es handelt sich dabei insbesondere um drei Aspekte:

a) Erstens eine dialogische und gewaltfreie Kommunikation, welche den Interaktionspartner nicht instrumentalisiert. Es ist der Entwurf einer Kommunikation, welche die Forderung des anderen danach, gehört zu werden, in zweifacher Hinsicht ernst nimmt. Erstens in Hinsicht darauf, die Möglichkeit zur Artikulation zu erhalten, und zweitens unter Verzicht auf epistemische Gewalt, welche in der instrumentellen Aneignung von Äußerungen besteht. ${ }^{42}$ Kommunikative Macht ${ }^{43}$ ist nach Arendt das, "was den öffentlichen Bereich, den potentiellen Erscheinungsraum zwischen Handelnden und Sprechenden, überhaupt ins Dasein ruft und im Dasein erhält «. 44

b) Zweitens setzt Arendt Menschen voraus, welche den Mut haben, zu offenbaren, ${ }^{45}$ wer sie sind, welche in einem öffentlichen Wettkampf zwischen sich wertschätzenden Personen um die Anerkennung ihrer Narrationen und Forderungen kämpfen. Es ist nicht die Kunst der Täuschung und der klugen Schachzüge, die

41 Joas 2011, S. 163; Joas 1999, S. 285.

42 Siehe hierzu De La Rosa 2012.

43 Das Wort » Macht « leitet sich, so Arendt, vom griechischen » dýnamis «, vom lateinischen »potentia « und im deutschen Sprachgebrauch von »möglich « ab. Es verweist damit auf einen möglichen Erscheinungsraum, in welchem Menschen zeigen können, wer sie sind, aber zugleich auch auf einen Raum für das Erscheinen von gemeinsamen politischen Prinzipien. Vgl. Arendt 2007 [1960].

44 Ebd., S. 252.

45 Ebd., S. 218 und 232. Zur Übersetzung des Begriffs der Offenbarung in den Bereich des Politischen siehe den Abschnitt »Die Einhegung der aneignenden Potenz durch Sakralisierung des menschlichen Lebens «.

Leviathan, 42. Jg., 2/2014 
hier zählt, sondern die Fähigkeit, die Karten offenzulegen. Sprechen und Handeln haben eine Aufschluss gebende Qualität, denn sie lassen das Einmalige einer Person, das Wer-einer-ist, sichtbar werden. ${ }^{46}$ Wo Menschen zeigen, wer sie sind, treten sie aus dem Konformismus der Gesellschaft heraus, kündigen die »Herrschaft des Niemands « 47 auf und nehmen eine ganz eigene Position im menschlichen »Bezugsgewebe « ein. Hierin liegt eine Ursache von Konflikten, welche jedoch durch die wechselseitige Anerkennung als Personen mit der legitimen Forderung, gehört zu werden, eingehegt wird.

c) Drittens setzt die Erfahrung der Selbsttranszendenz die konkrete Anwesenheit der Menschen vor Ort voraus. Sie kann nicht medial übertragen oder vermittelt werden. »Für eine vernünftige Meinungsbildung bedarf es des Meinungsaustauschs, um sich eine Meinung zu bilden, muß man dabei sein; und wer nicht dabei ist, hat entweder -im günstigsten Falle - gar keine Meinung, oder er macht sich in den Massengesellschaften des neunzehnten und zwanzigsten Jahrhunderts aus allen möglichen, konkret nicht mehr gebundenen Ideologien einen Meinungsersatz zurecht. «48 Das Dabeisein ist deshalb von Bedeutung, weil es die Erfahrung der Selbsttranszendenz im Bereich des Politischen ermöglicht. Denn so wenig wie eine Radiosendung die Anwesenheit bei einem Gottesdienst ersetzen kann, wenn es um die Erfahrung der Selbsttranszendenz geht, ebenso wenig kann eine TV-Show Erfahrungen der politischen Selbsttranszendenz generieren.

Arendt überträgt die religiös konnotierte Erfahrung der Selbsttranszendenz in den politischen Bereich, indem die öffentliche Sphäre zum zentralen Ort der politischen Erfahrung wird. Der öffentlich ausgetragene dialogische Wettkampf um Artikulationen und Werte zwischen sich anerkennenden politischen Akteuren wird so für die Akteure selbst und für ihr Publikum zu einem Ort möglicher Selbsttranszendenz. Auf diesem Wege nimmt Arendt eine Aneignung religiöser Erfahrungen und Gehalte vor und weist ihnen einen Ort im Bereich des Politischen zu. Das Ziel dieser Aneignung ist eine säkulare Vergemeinschaftung. Diese basiert auf politischen Erfahrungen der Selbsttranszendenz, welche im dialogischen Wettkampf oder im Beiwohnen eines solchen Wettkampfs zwischen sich als einzigartig anerkennenden Individuen gemacht werden können. Selbsttranszendenz wird hier zu einer grundlegenden politischen Kategorie, weil sie nicht mehr auf etwas Göttliches gerichtet ist, sondern als intersubjektive Erfahrung gedeutet wird, welche, so lässt sich mit Joas sagen, Gefühle subjektiver Evidenz und affektiver Intensität in Bezug auf politische Prinzipien erzeugt, welche nur in der Gemeinschaft mit anderen Menschen erfahrbar sind.

Als problematisch ist daran die Einschränkung auf den politischen Bereich zu werten. Während Joas die Möglichkeit von »erschütternden« Erfahrungen auch außerhalb des Bereichs des Politischen aufzeigt, erscheint die Praxis der Sakralisie-

46 Vgl. Arendt 2007 [1960].

47 Ebd., S. 51.

48 Arendt 2006 [1965], S. 303. 
rung bei Arendt allein als ein politisches Handeln. »Erschütternde« Erfahrungen sind für Arendt deshalb nur relevant, wenn sie im Bereich des kommunikativen Handelns und damit im Bereich des Politischen stattfinden.

\section{Die Sakralisierung des Menschen als Praxis der (Selbst-)Heiligung}

Als zweites den Agon einhegendes Element kann die Sakralisierung des menschlichen Lebens genannt werden. Die Sakralisierung, also die Heiligung, war zunächst ein rein religiöser Akt, welcher die Abtrennung von Dingen oder Personen, also ihre Vorrangstellung gegenüber anderen, hervorhob. Arendt eignet sich diese Art der Auszeichnung an und überträgt sie in den politischen Bereich als eine Praxis der (Selbst-)Heiligung. Sie entfernt dabei den Leistungsgedanken, indem sie die Sakralisierung qua Person, also qua Menschsein vornimmt und der physischen wie seelischen Unversehrtheit jedes Einzelnen den Vorrang vor allem anderen einräumt. Nicht die Gattung, der Staat, die Nützlichkeit oder die Gemeinschaft sind das Maß aller Dinge, sondern die Unversehrtheit des Einzelnen. Diese kann jedoch nur durch ein ständiges Ringen geschützt werden, denn eine unverfügbare oder außerpolitische Kategorie bietet Arendts Begriffsgebäude nicht. Es lässt vielmehr sichtbar werden, dass nicht nur auf der Ebene politischer Entscheidungen um die Sakralisierung der Person gerungen werden muss, sondern auch auf der Ebene der politischen Theorie und der Geschichtsschreibung.

\section{Aneignung als Stilmittel politischer Theorie}

Arendt ist immer wieder vorgeworfen worden, sie mache die griechische Polis zu einem Vorbild, welches diesen Vorbildcharakter, den Arendt zeichnet, niemals besessen habe. Dieser Vorwurf, so möchte ich argumentieren, trifft Arendt nicht, weil die Aufgabe der politischen Theorie für sie darin liegt, wie die Gründerväter im Falle der Amerikanischen Revolution "aus der Vergangenheit Sinn herauszulesen $« .{ }^{49}$ Aufgabe der politischen Theorie ist es also nicht, nach wahren Begebenheiten in der Geschichte zu suchen, sondern aus der Geschichte Sinnelemente zu gewinnen und sie in neue Narrationen einzubinden, das heißt sich die Geschichte anzueignen, um der Gegenwart Sinn zu geben. Denn Sinn ist es, woran es dem Herstellen und damit der Politik der Notwendigkeit mangelt. Weil sich das Streben nach der Herstellung einer Dingwelt in einer infiniten Zweck-Mittel-Schlaufe verfängt, wird alles, was zunächst Zweck der Anstrengung war, zum Mittel für einen anderen Zweck. Der Nutzen als End- oder Selbstzweck ist daher sinnlos, weil das Ideal des Nutzens nicht mehr damit erklärt werden kann, dass es nützlich sei, »über seinen eigenen Nutzen und Zweck befragt, muß es die Auskunft verweigern « ${ }^{50}$ Denn es gibt keine Antwort auf die Frage, was der Nutzen des Nutzens ist.

Der politischen Theorie fällt daher die Aufgabe zu, an der Erzeugung von Sinn zu arbeiten. Sie ist dabei jedoch selbst auf Prozesse kommunikativer Macht angewie-

49 Arendt 2006 [1961], S. 3-15.

50 Arendt 2007 [1960], S. 183.

Leviathan, 42. Jg., 2/2014 
sen, in welchen es ihr gelingen muss, die politischen Prinzipien, die sie zu transportieren versucht, verständlich und erfahrbar zu machen. Ich werde daher im Folgenden argumentieren, dass Arendt ein ganz bestimmtes theoretisch-konzeptionelles Mittel einsetzt, um » um Zustimmung zu werben ${ }^{51}$ - die Sakralisierung des menschlichen Lebens.

\section{Die Einhegung der aneignenden Potenz durch Sakralisierung des menschlichen Lebens}

Der Begriff der Sakralisierung, wie ihn Hans Joas in Die Sakralität der Person verwendet, basiert auf der Beobachtung eines Prozesses: »[...] in dem jedes einzelne menschliche Wesen mehr und mehr und in immer stärker motivierender und sensibilisierender Weise als heilig angesehen und dieses Verständnis im Recht institutionalisiert wird «.52 Arendt befördert diese Sakralisierung der Person, indem sie Eigenschaften Gottes wie die Fähigkeit zur Schöpfung, zum Neuanfang oder des Verzeihens auf den Menschen überträgt und so das menschliche Leben durch die Annäherung an das Göttliche sakralisiert. Der Mensch besitzt nach Arendt einerseits die Fähigkeit, bisher Gewesenes oder Getanes hinter sich zu lassen und Neues zu beginnen. Die Gefahr, die mit dem Neu-Anfangen verbunden ist, nämlich die Unabsehbarkeit dessen, was auf einen Neuanfang folgt, kann durch die zweite göttliche Fähigkeit des Menschen, die des Verzeihens, aufgefangen werden. »Das Heilmittel gegen Unwiderruflichkeit - dagegen, dass man Getanes nicht rückgängig machen kann, obwohl man nicht wusste, und nicht wissen konnte, was man tat - liegt in der menschlichen Fähigkeit zu verzeihen. «53 Das Verzeihen zielt einerseits auf Getanes, bereits Geschehenes, das heißt Beziehungen etwa nachfolgender Generationen sollen nicht mehr belastet werden durch das, was vorher geschehen ist, sie sollen in der Lage sein, einen neuen Anfang zu setzen. Gleichzeitig erleichtert das Verzeihen den Neuanfang insofern, als es die Angst vor unverzeihlichen Fehlern minimiert, denn es ist nicht absehbar, wohin ein Neuanfang sich im menschlichen Bezugsgewebe entwickelt.

Arendt überträgt so die Fähigkeit, Sünden zu verzeihen, auf den Menschen. Verzeihen ist, so Arendt, keine religiöse Erfahrung, sondern eine genuin politische, weil es die einzige Möglichkeit ist, den »Schaden, den alles Handeln unweigerlich mitanrichtet " ${ }^{54}$ einzuhegen, indem es sich in das Gewebe menschlicher Beziehungen einmischt, ohne alle Konsequenzen absehen zu können. »Nur durch dieses dauernde gegenseitige Sich-Entlasten und Entbinden können Menschen, die mit der Mitgift der Freiheit auf die Welt kommen, auch in der Welt frei bleiben [...].« ${ }^{55}$

51 Arendt 2006 [1979], S. 459.

52 Diese Art der Sakralisierung, so Joas, ist nicht auf religiöse Bedeutungen beschränkt, sondern auch säkulare Gehalte können »die Qualität annehmen, die für die Sakralität charakteristisch sind; subjektive Evidenz und affektive Intensität «. Joas 2011, S. 18.

53 Arendt 2007 [1960], S. 301.

54 Ebd., S. 305.

55 Ebd., S. 306. 
Der Mensch emanzipiert sich von Gott, wird ihm in seinen Fähigkeiten zur Schöpfung einer (Ding- beziehungsweise sozialen) Welt, seiner Fähigkeit zum Neuanfang und zum Verzeihen ähnlich und damit zugleich auch heilig. Die Übertragung lässt sich gut an einem Augustin-Zitat aus der Vita activa aufzeigen:

"Damit ein Anfang sei, wurde der Mensch geschaffen, vor dem es niemand gab [...] « Dann Arendt weiter: "[S]eine Erschaffung ist nicht der Beginn von etwas [...] sondern das Anfangen eines Wesens, das selbst im Besitz der Fähigkeit ist anzufangen. [...] Mit der Erschaffung des Menschen erschien das Prinzip des Anfangs, das bei der Schöpfung der Welt noch gleichsam in der Hand Gottes und damit außerhalb der Welt verblieb, in der Welt selbst und wird ihr immanent bleiben, solange es Menschen gibt. «56

Arendt eignet sich auch den Begriff der Offenbarung an und verweist damit auf die Offenbarung Jesu Christi, in welcher es darum geht, den göttlichen Heilsplan verständlich zu machen. In den Bereich des Politischen übersetzt, wird daraus die Forderung, die eigenen Handlungen für andere transparent zu machen, damit sie sich entscheiden können, ob sie dem vorgeschlagenen »Heilsplan « folgen wollen oder nicht. Es wird keine blinde Folgebereitschaft verlangt oder durch Tricks und Kniffe Folgebereitschaft »ergaunert ", sondern es wird aufrichtige Überzeugungsarbeit geleistet, sodass jeder, der folgt (im Rahmen des Vorhersehbaren), weiß, wem und was er folgt.

\section{Fazit}

Arendt übersetzt die religiösen Begriffe in die Sprache der politischen Theorie und eröffnet damit den Blick auf eine mögliche Vergemeinschaftungsform, welche säkular ist und zugleich religiöse Bedeutungsgehalte und Praktiken zugunsten einer zutiefst demokratischen Vergemeinschaftungsform zugänglich macht. Die Wertschätzung der Pluralität und jedes Einzelnen in seiner Einzigartigkeit bleibt in ihrer Konzeption nicht allein der Institution des Rechts überlassen, denn Rechte werden von Staaten garantiert, die im Moment der größten Not gerade nicht imstande oder bereit sind, diese Rechte zu garantieren. ${ }^{57}$ Daher argumentiert Arendt, dass es einer anderen Verortung des Respekts vor dem menschlichen Leben bedarf, als es in einer Konzeption positiver Rechte aufzuheben. Diese Verortung findet sich bei Arendt in den dialogisch-demokratischen Formen der Interaktion, also im Miteinander-Sprechen und -Handeln, als auch in der Sakralisierung des menschlichen Lebens sowie der Erfahrbarkeit von Werthaftigkeit durch politische Erfahrungen der Selbsttranszendenz wieder. Die Unversehrtheit des Einzelnen inklusive seiner Fähigkeit, sich zu artikulieren, ist das Maß aller Dinge, aber dieser Maßstab muss immer wieder neu gesetzt und erkämpft werden. Ein Ja zu diesem Maßstab muss immer wieder das Ergebnis von Auseinandersetzungen um die und mit der Vergangenheit sowie des Blicks auf eine mögliche Zukunft sein. Der politische Wettkampf, welcher diese Bezeichnung verdient, weil er im Sinne Arendts dialogisch ist, wird einerseits von den dialogisch-demokratischen Formen des Handelns und andererseits von den

56 Ebd., S. 216.

57 Vgl. Arendt 2009 [1955]; hierzu auch Birmingham 2006.

Leviathan, 42. Jg., 2/2014 
Erfahrungen der Selbsttranszendenz und der Sakralisierung des menschlichen Lebens eingehegt. Die Erfahrungen der Selbsttranszendenz, die im diskursiven Miteinander gemacht werden, bilden den sozialen Kitt, welcher den Bestand der dialogisch-umbildenden Narrationen sichert und mit Leben erfüllt. Bei dieser Art von Auseinandersetzung können Werte entstehen, weil Menschen hier die Erfahrung machen können, was es bedeutet, sich für eine als wertvoll erfahrene Sache gemeinsam einzusetzen. Zugleich sollte aber klar sein, dass ein »kategorischer Schutz der Person « oder eines anderen Wertes, wie ihn Joas mit einer außerpolitischen Kategorie zu schaffen sucht, ${ }^{58}$ mit Arendt nicht begründet werden kann. Dafür sind die Ideen der Kontingenz und der Aneignung in ihrem Theoriegebäude zu präsent. Der Schutz der Person muss mit Arendt immer wieder neu diskursiv hergestellt und bestätigt werden - nicht zuletzt mit den konzeptionellen Mitteln der politischen Theorie. Daher greift Arendt auf religiöse Bedeutungsgehalte und Praktiken zurück, deutet diese um und macht sie für den politischen Kontext fruchtbar, indem sie uns die konstruktiven Wirkungen aufzeigt, welche die im religiösen Kontext erlernten Praktiken etwa des Verzeihens oder der Selbsttranszendenz nach ihrer Umdeutung auch im politischen Kontext entfalten können.

\section{Literatur}

Alexander, C. Jeffrey 2011. Performance and power. Cambridge: Polity Press.

Allen, Amy 1999. "Solidarity after identity politics: Hannah Arendt and the power of feminist theory ", in Philosophy and Social Criticism 25, 1, S. 97-118.

Arendt, Hannah 2003 [1929]. Der Liebesbegriff bei Augustin. Berlin, Wien: Philo.

Arendt, Hannah 2006 [1961]. Between past and future. London: Penguin.

Arendt, Hannah 2006 [1965]. Über die Revolution. München, Zürich: Piper.

Arendt, Hannah 2006 [1979]. Vom Leben des Geistes. München, Zürich: Piper.

Arendt, Hannah 2007 [1960]. Vita activa oder vom tätigen Leben. München, Zürich: Piper.

Arendt, Hannah 2007 [1993]. Was ist Politik? Fragmente aus dem Nachlaß, hrsg. v. Ludz, Ursula. München, Zürich: Piper.

Arendt, Hannah 2009 [1955]. Elemente und Ursprünge totaler Herrschaft. München, Zürich: Piper.

Birmingham, Peg 2006. Hannah Arendt and human rights. The predicament common responsibility. Bloomington, Indianapolis: Indiana University Press.

Burkhardt, Johannes 2009. Deutsche Geschichte in der Frühen Neuzeit. München: C. H. Beck.

Canal, Héctor et al. 2013. Das Heilige (in) der Moderne. Denkfiguren des Sakralen in Philosophie und Literatur des 20. Jahrbunderts. Bielefeld: transcript.

De La Rosa, Sybille 2012. Aneignung und interkulturelle Repräsentation. Wiesbaden: Springer VS.

Frank, Martin 2001. »Hannah Arendts Begriffe der Weltentfremdung und Weltlosigkeit in Vita activa im Lichte ihrer Dissertation Der Liebesbegriff bei Augustin «, in » The Angel of History is looking back «. Hannah Arendts Werk unter politischem, ästhetischem und historischem Aspekt, hrsg. v. Neumann, Bernd et al., S. 127-151. Würzburg: Königshausen und Neumann.

Gotthard, Axel 2002. "Strukturkonservativ oder aggressiv? Die geistlichen Kurfürsten und der Ausbruch des teutschen Konfessionskriegs ", in Friedliche Intentionen - kriegerische Effekte. Studien zur neueren Geschichte 1, hrsg. v. Schulze, Winfried, S. 140-168. St. Katharinen: Scripta Mercaturae.

58 Vgl. Joas 2011, S. 107. 
Greven, Michael 1999. Die politische Gesellschaft. Opladen: VS Verlag für Sozialwissenschaften.

Habermas, Jürgen 1991. Erläuterungen zur Diskursethik. Frankfurt a. M.: Suhrkamp.

Habermas, Jürgen 1995. Theorie des kommunikativen Handelns. Zur Kritik der funktionalistischen Vernunft, Band 2. Frankfurt a. M.: Suhrkamp.

Habermas, Jürgen 2012. Nachmetaphysisches Denken II. Berlin: Suhrkamp.

Holzinger, Markus 2007. Kontingenz in der Gegenwartsgesellschaft. Dimensionen eines Leitbegriffs moderner Sozialtheorie. Bielefeld: transcript.

Joas, Hans 1999. Die Entstehung der Werte. Frankfurt a. M.: Suhrkamp.

Joas, Hans 2009. » Die säkulare Option. Ihr Aufstieg und ihre Folgen «, in Deutsche Zeitschrift für Philosophie 57, 2, S. 293-300.

Joas, Hans 2011. Die Sakralität der Person. Eine neue Genealogie der Menschenrechte. Berlin: Suhrkamp.

Schilling, Heinz 2007. Konfessionalisierung und Staatsinteressen 1559-1660. Handbuch der Geschichte der Internationalen Beziehungen, Band 2. Paderborn: Schöningh.

Schönherr-Mann, Hans-Martin 2006. Hannah Arendt. Wabrheit, Macht, Moral. München: C. H. Beck.

Villa, Dana 1992. »Beyond good and evil. Arendt, Nietzsche, and the aestheticization of political action", in Political Theory 20, 2, S. 274-308. 
Zusammenfassung: Das Hauptargument des Beitrags lautet, dass Arendt an der Entstehung von Werten arbeitet, indem sie Praktiken wie das Erschaffen, Verzeihen, Sakralisieren aus dem religiösen Zusammenhang löst und radikal politisch deutet. Sie unterscheidet sich darin von Hans Joas, welcher mit der Sakralisierung der Person nach einer Möglichkeit sucht, den Schutz der Person dem Politischen zu entziehen und ihr via Heiligkeit einen unverfügbaren Charakter zu geben, denn bei Arendt bleibt die Heiligkeit der Person immer dem Spiel der Kontingenz ausgesetzt und von Aushandlungen abhängig.

Stichworte: Säkularisierung, Sakralisierung, Selbsttranszendenz, Politisierung

\section{Hannah Arendt in between secularization and sacralization}

Summary: While Hans Joas argues for a sacralization of the person which withdraws the protection of the person from the political, it is maintained that Arendt's writing proposes a sacralization that does not negate the contingency of sacralizations and rather assumes it to be part of human freedom. Furthermore it is argued, that although Arendt appropriates religious terms and practices, she subordinates them, according to her understanding of emancipation, to the political, and in this way develops the model of a secular form of collectivization.

Keywords: contingency, secularization, sacralization, political self-transcendence

Autorin

Dr. Sybille De La Rosa

Institut für Politische Wissenschaft

Lehrstuhl für Moderne Politische Theorie

Bergheimer Straße 58

69115 Heidelberg

sybille.delarosa@ipw.uni-heidelberg.de 